\title{
Unusual Light Curves of Some Carbon Stars
}

\author{
ANDREJS ALKSNIS \\ Radioastrophysical Observatory, Riga, Latvia
}

Among more than 200 carbon stars monitored photographically with the Baldone Schmidt telescope for time intervals from several years to nearly 3 decades in two to five passbands, three large-amplitude variable stars with exceptional properties of light variation were noticed.

The cyclic component of the light variation of RW LMi $=$ CIT 6 in blue light (cycle length between 350 and $600 \mathrm{~d}$ ) is entirely different from that in the red and infrared (long-period variation typical for carbon stars: $P=605 \mathrm{~d}$ before $1988, P=628 \mathrm{~d}$ afterwards). A secondary variation with a probable cycle length of about $8 P$, however, is shared by both passbands, as are the shorter-timescale $(3-30 \mathrm{~d})$ fluctuations. What causes the cyclic blue light variation of RW LMi?

For DY Per, both a long-period (however rather irregular) variation with a cycle length of $792 \mathrm{~d}$ and deep light declines similar to those observed in $\mathrm{R}$ Coronae Borealis variables have been found. The last five decline events of the RCB type occurred with time intervals of $930,770,730$ and $680 \mathrm{~d}$, not very different from the cycle length. Is DY Per a long-period variable or an $\mathrm{RCB}$ variable, or both?

In AFGL $2881=$ V366 Lac, a long-period variation of the Mira type $(P=$ $562.5 \mathrm{~d}$ ) is superimposed upon a secondary variation of extraordinarily large amplitude, unprecedented for a carbon star (5 mag in red light). From an optically recognizable carbon star during the years 1979-1996, the star has changed to an infrared carbon star.

More information on these stars is published in or submitted to Baltic Astronomy as well as submitted to Astronomical and Astrophysical Transactions. 